\title{
Preparation and Prospect of Polymer Nanocomposites Used in High Voltage Equipment
}

\author{
Xin-Na ZHANG ${ }^{1,2, a, *}$ and Cheng-Li LIU ${ }^{1, b}$ \\ ${ }^{1}$ Faculty of Electrical Engineering, Kunming Metallurgy College, Kunming Yunnan 650033, China \\ ${ }^{2}$ Faculty of Electrical Power Engineering, Kunming University of Science and Technology, \\ Kunming 650500, China \\ a1821243365@qq.com, b1251952379@qq.com \\ *Corresponding author: Xinna Zhang
}

Keywords: Polymer nanocomposite, High voltage equipment, Preparation process, Prospect, Properties

\begin{abstract}
Recently, in the field of high voltage equipment, polymer nanocomposites have received increasing attentions, due to their excellent electrical properties, such as high breakdown strength and high resistance to partial discharges, and their good mechanical and thermal properties, such as high mechanical strength and high heat durability and so on. In this article, the main preparation methods of polymer nanocomposites, including of blending method, intercalation compound method, in-situ polymerization method and sol-gel method, are reviewed, and the merits and faults of different methods are discussed.
\end{abstract}

\section{Introduction}

At present, polymers have used widely in electrical fields due to their excellent properties, such as high dielectric permittivity, low density and low cost and so on [1-2]. However, with the rapid development of high voltage equipment, more and more problems of traditional polymers used in this field have been exposed, such as the low dielectric strength and insulation aging and so on.

Recently, polymer nanocomposites have received increasing attentions in the field of high voltage equipment, due to their excellent electrical properties, such as high breakdown strength and high resistance to partial discharge, and their good mechanical and thermal properties, such as high mechanical strength and high heat durability and so on. A large number of works have confirmed that the inorganic nanoparticles filled in polymers can improve significantly their electrical insulation properties, for example the resistivity of polymer materials is increased and the dielectric of polymer materials loss is reduced [3-5]. Tanaka et al. [6] prepared Cross-linked Polyethylene nanocomposites and reported the evidence of its enhanced dielectric properties. Shah et al. [7] studied the preparation process and dielectric strength of HDPE clay nanocomposites, and found that the dielectric strength was improved by the nanocomposites. Tanaka et al. [8] investigated the properties of epoxy/SiC and found, with the increase of nano-filler, the erosion depth of epoxy/SiC decreased.

In this present work, the preparation methods of polymer nanocomposites are reviewed, the corresponding flowcharts of preparation process are presented, and the merits and defects of different processes are discussed.

\section{Blending Method}

Blending method is the most direct and simple one for producing polymer nanocomposites, which is achieved by fully mixing of nanoparticles and polymer matrix $[9,10]$. This kind of method can be divided into two ways, solution blending and emulsion blending. The difference between them lies mainly in that the solution used in the production is different. Fig.1 shows the general process to prepare polymer nanocomposites by blending method. Firstly, to decrease the gravitational potential energy, the nanoparticles are surface treated by chemical or physical method, for example surfactant 
treatment. Then the treated nanoparticles, polymer matrix and a certain amount of medium materials (for example water) are put into a container and fully mixed. The obtained mixture is stood for a period of time. The end, the stood mixture is filtered to obtain the final products.

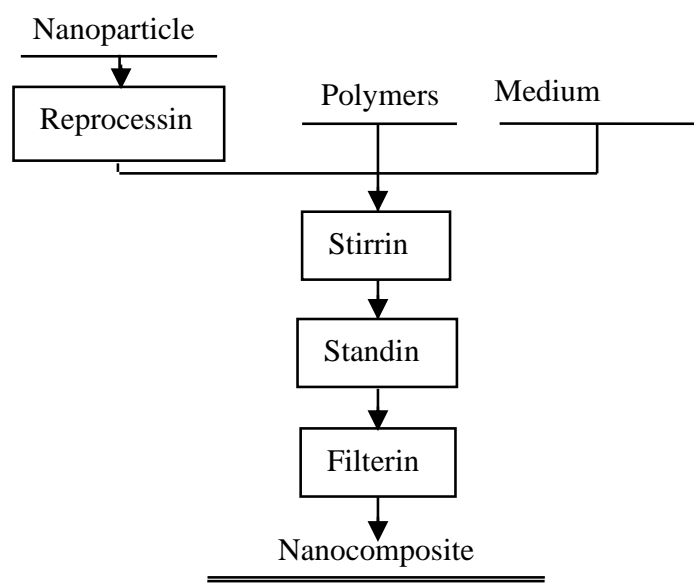

Fig.1 Flow chart of blending method

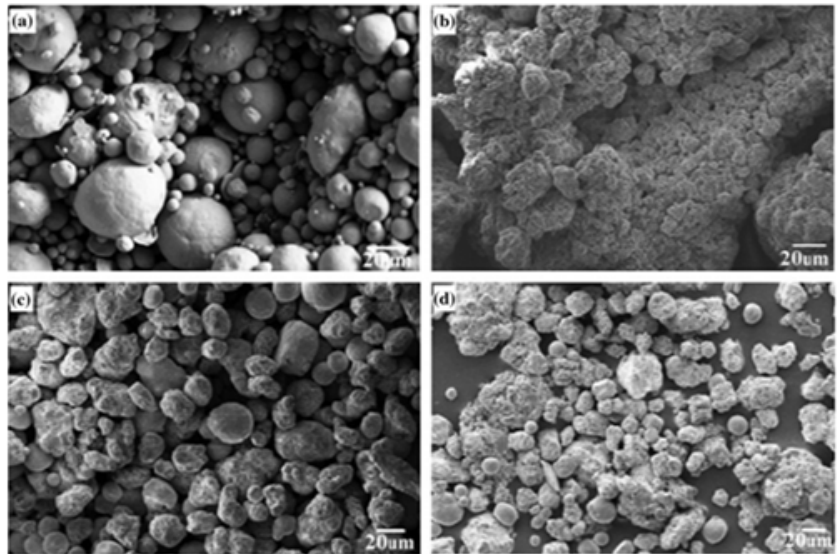

Fig.2 SEM images of Cu/PEO composite [12]

In recent years, many researches have prepared polymer nanocomposites by this process and studied their related properties. Zhou et al. [11] investigated EPEI/HSi-TiO2-hybridized resins and found that this kind of materials could provide outstanding corona resistance, high breakdown strength and remarkably decreased partial discharge. Azizurrahaman et al. [12] prepared $\mathrm{Cu}$ /polyethylene oxide composite $(\mathrm{Cu} / \mathrm{PEO})$ by mechanical blending method. The SEM microphotographs of the $\mathrm{Cu} / \mathrm{PEO}$ composites with different filler ratio are show in Fig.2. Form Fig .2(c)-(d), it can be seen that the $\mathrm{Cu}$ particles are well dispersed into the PEO matrix, which can produce a big change into the interfacial polarization leading to the higher dielectric properties.

The advantages of blending method lie in that it is easy to operate and realize industrialization. In addition, the shape and size of the filled nanoparticles can be selected and controlled easily. However, there are still many problems on the use of blending method in preparing polymer nanocomposites, such as the physical and mechanical methods are usually used to disperse the nanoparticles, however the great interface free energy of nanoparticles makes them easy to agglomerate.

\section{Intercalation compound method}

At present, intercalation compound is the primary method for producing polymer nanocomposites, which is achieved by inserting macromolecules, such as organic molecules, polyethylene epoxy and polyvinyl alcohol into the space between layers of inorganics with a layer structure on a nanoscale and treated by intercalating agent though ion-changed reaction $[13,14]$. The nanocomposites prepared by this kind of method have a distinctive structure feature, namely, the alternative organic layer and inorganic layer. In the inorganic matrix, the inserting approaches of organics includes: monomers intercalation and in-situ polymerization, solution intercalation, and melts intercalation. The process of preparing polymer nanocomposites by intercalation compound method is shown in Fig.3. First, polymers or monomers are inserted into the space between layers of inorganics. Then, under the thermal effects, the layer structures are destroyed by in-situ polymerizing of monomers intercalated in the crystal layers, intercalating polymer solution into tunnels between layers, or direct intercalating polymer melts into the space between layers, which makes the distance between layers increase and even dissociate. So, the polymer nanocomposites with the nanoscale dispersion of inorganic fillers in the polymer matrix are prepared finally. 


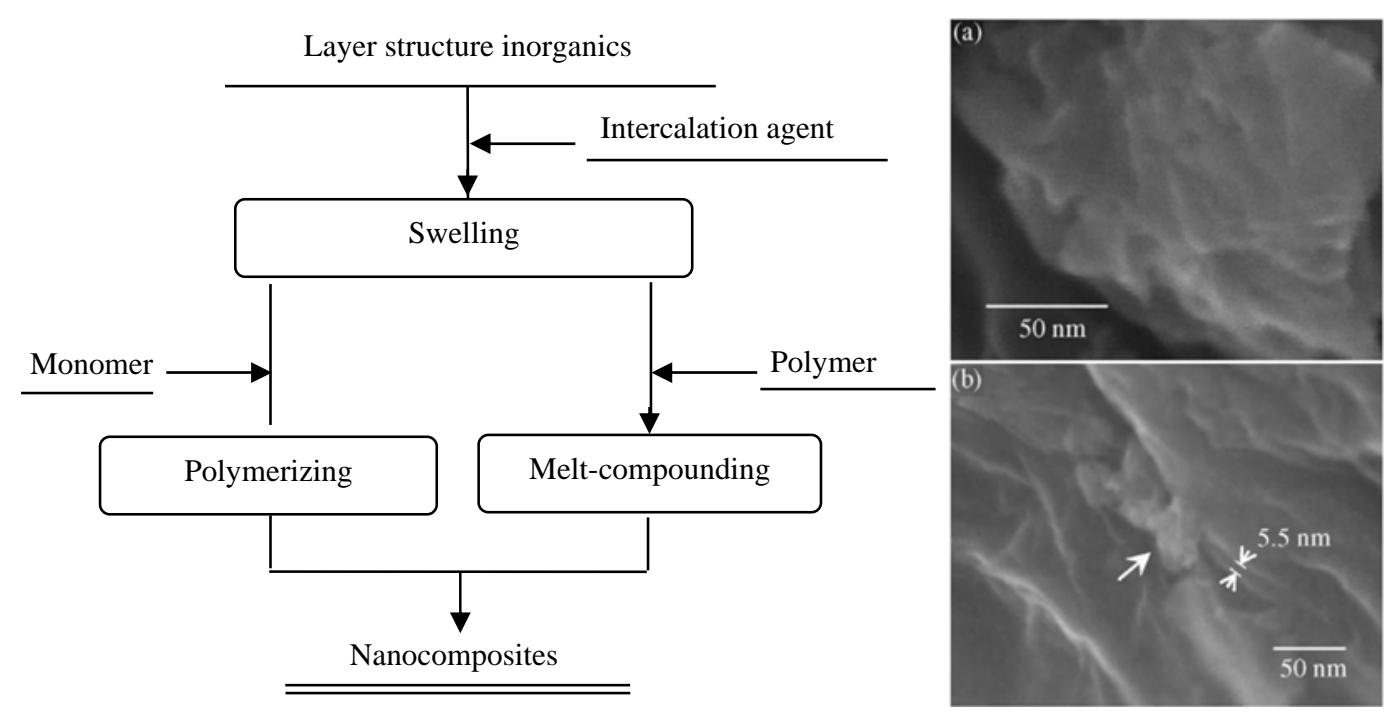

Fig.3 Flow chart of intercalation method

Fig.4 SEM images of GOC16Ti/S [16]

The inorganics used in intercalation compound method are some special compounds with layer structure, for example montmorillonite. MPS 3 (M represent bivalent metals, such as Mn, Cd, Zn and $\mathrm{Fe}$ and so on) is a good choice for the intercalation compound in the space of layers as flaky compounds [14]. Mallakpour et al [15] prepared PEI/OMMT nanocomposites by solution intercalation method, and they found the thermal property of materials strongly related to the dispersion of nanoclay in the polymer matrix. Chu et al. [16] investigated Synthesis of nanoporous graphite-derived carbon/ $/ \mathrm{TiO}_{2}-\mathrm{SiO}_{2}$ composites, and the SEM images of the composites prepared by intercalation compound method as shown in Fig.4.

Compositing to the matrixes, not only the mechanical properties of materials prepared by this method are increased significantly, but the composite materials have many especial performance, such as thermal stability, magnetism, electricity and nonlinear optics and so on [17]. But, there are still some limitations in the application of this method, such as melt intercalation method, the nanoparticles are still difficult to be dispersed uniformly. In addition, in this method, inorganics, for example organoclay, and high polymers must be able to be dissolved in same solvent, however, it is difficult for a lot of high polymers [14].

\section{In-situ dispersion polymerization method}

In-situ dispersion polymerization is the most representative preparation method for polymer nanocomposites $[18,19]$. The process of preparing polymer nanocomposites by this kind of method is shown Fig.5. First, nanoparticles are surface treated by coupling agent. Then, the treated nanoparticles are dispersed uniformly into polymer monomer solution by means of mechanical blending or ultrasonic dispersion and so on. Thirdly, the polymerizations of monomer are caused in the nanoparticle surfaces, to thereby form polymer nanocomposite.

In recent years, many researches have prepared nanocomposites materials by this method. Ren et al. [20] prepared Fe/ER nanocomposites and investigated the dielectric properties, and the results found that the materials had excellent comprehensive properties. Xie et al. [21] prepared polypyrrole/nano $\mathrm{SiO}_{2}$ composite and investigated the properties of electromagnetic absorption. The results showed that $\mathrm{SiO}_{2}$ nanoparticles dispersed uniformly in the materials, as shown in Fig.6. In addition, the advantages of this method lies in the conditions is mild, the nanoparticles are dispersed uniformly, and the properties of nanoparticles is remained.

In-situ dispersion polymerization method has a broader and milder reaction conditions. The environment of polymerization can be aqueous or oil phase, the monomers can carry out radical polymerization or polycondensation reaction, especially, there is no need for additional thermal process. Moreover, the polymer monomers have smaller molecules, lower viscosity and have been surface modification. So, in the matrix materials, the nanoparticles are dispersed easily and the 
nano-properties is still intact. Due to not need heat treatment, the resulting degradation is avoided. Therefore, the uniformity of system and the stable basic properties are retained. This preparation method can be applied to most of polymer matrix organic-inorganic nanoparticles. However, the method is still limit in the practical application due to it only applies to colloidal particles including metals, sulfides and hydroxides which can makes the monomers in-situ polymerize in the solution to prepare the needed nanocomposites.

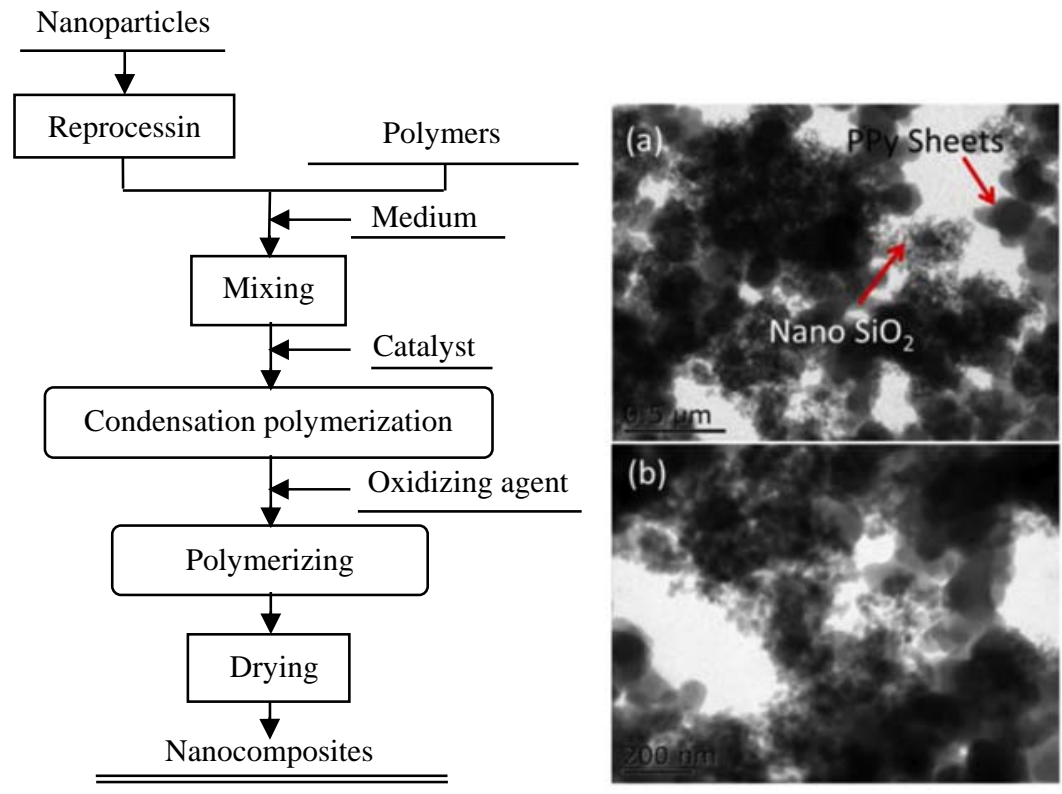

Fig.5 Flow chart of In-situ dispersion polymerization

Fig.6 (a, b) TEM images of 3D-PPy/20\% $\mathrm{SiO}_{2}[21]$

\section{Sol-gel method}

Sol-gel is an important method for producing polymer nanocomposites. The organic-inorganic nanocomposites prepared by sol-gel method began in 1980s. It is well known, the sol-gel reaction is a method to prepare inorganics, such as the precursors of glasses or ceramics, at relatively low temperatures. The reaction is generally divided into two steps: hydrolysis of metal alkoxides to produce hydroxyl groups, followed by polycondensation of the hydroxyl groups and residual alkoxyl groups to form a three-dimensional network [22]. The general process can be represented in Fig 7. The sol-gel process generally starts with alcoholic or other low molecular weight organic solutions of monomeric, metal or semimetal alkoxide precursors. Generally, both the hydrolysis and condensation reactions occur simultaneously once the hydrolysis reaction has been initiated to generate low molecular weight byproducts such as alcohol and water. The general process as following: First, the precursors such as siloxane and the polymer are dissolved in water or organic solvent to form a homogeneous solution. Second, Hydrolysis and condensation of precursors occur to form sol. Finally, the composite materials can be prepared by volatile solvents or drying process to prepare gel with network structure [23,24].

In the past years, amount of researches have been carried out on the preparation of nanocomposites materials. Kuan et al. [25] prepared PMMA/VTES-g-EG composites by this method and studied its thermal stability. Ma et al. [26] investigated the preparation of $\mathrm{PI} / \mathrm{SiO}_{2}$, the SEM images of $\mathrm{PI} / \mathrm{SiO}_{2}$ composite particles by this method are shown in Fig. 8.

The advantages of this method lie in that high purity and uniformity, low sintering temperature, reaction easily controlled and good forming and so on. However, there are still some limitations in the process of actual application, such as most of precursors are expensive and toxic. It is difficult to prepare crystalline composites including polymers and inorganic oxide nanoparticles due to polymers are unaffordable general the high temperature, which is the necessary condition to make inorganic nanoparticles form crystal structure, and the process of sol-gel usually needs a few days or weeks. Especially, the contractionary brittle fracture due to the volatile of solvents, small molecules and water is the biggest question for this method. 


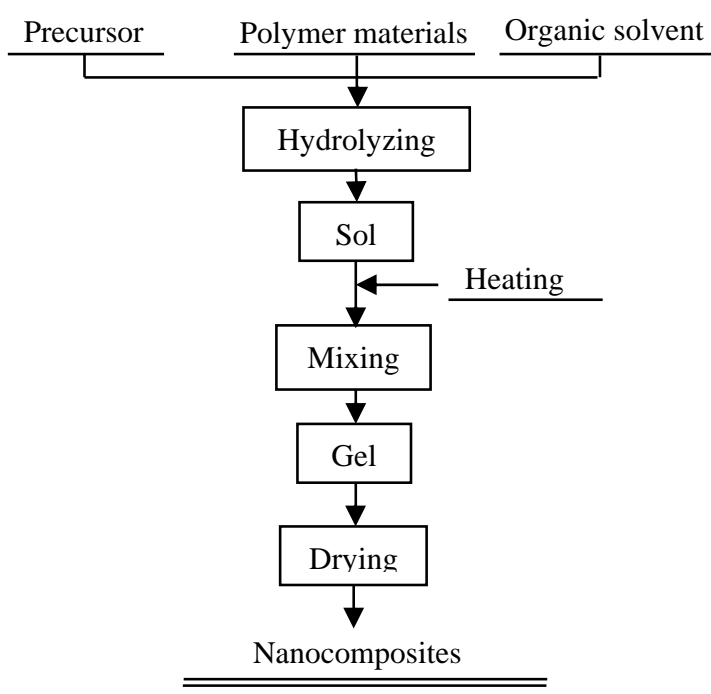

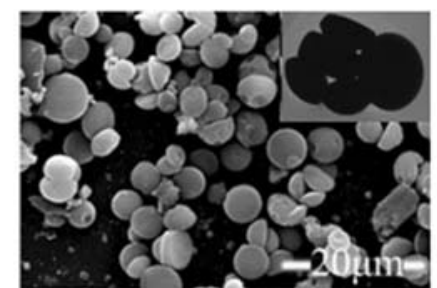
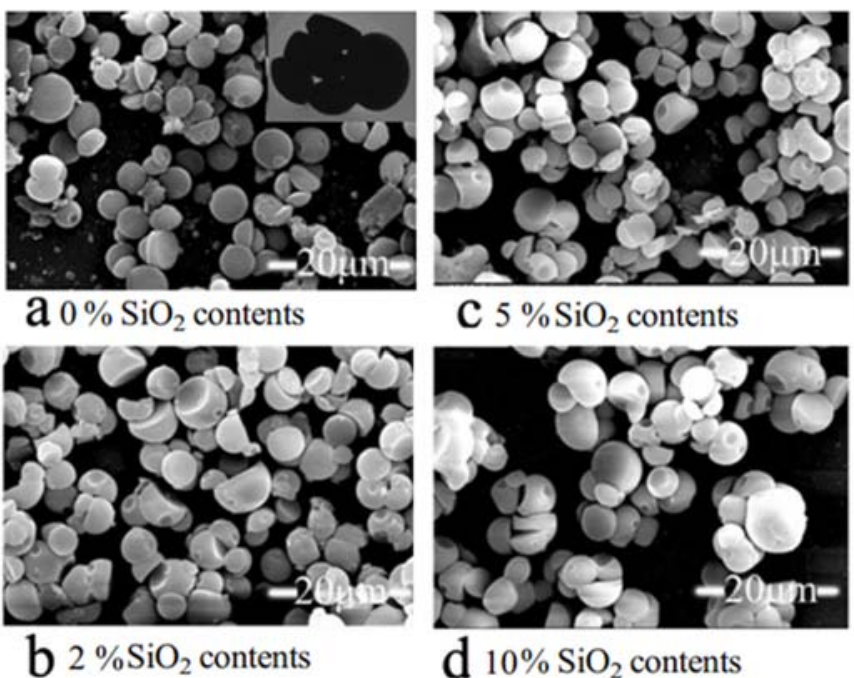

C $5 \% \mathrm{SiO}_{2}$ contents

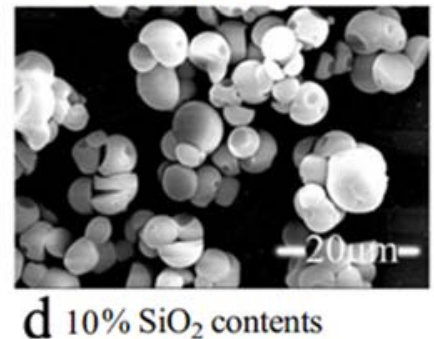

Fig.7 Flow chart of sol-gel method

Fig.8 (a, b) SEM images of PI/SiO2 composite particles.[26]

\section{Prospect}

At present, the main ways for preparing polymer nanocomposites include the above mentioned four methods. There are different characteristics and application range for each method respectively. Besides various electrical properties, polymer nanocomposites have been extensively investigated on the high voltage insulation application. The results have shown the promising of this kind of materials in insulation perspectives field [27,28]. In addition, the application of high density polyethylene nanocomposites in electrical insulation perspectives has been investigated deeply, and is found that it has excellent dielectric strength, volume resistivity and surface resistivity [7]. As an important material of the main insulation in high voltage equipment, epoxy nanocomposites have good space charge behavior, PD resistance and dielectric strength [29,30]. All of these have confirmed polymer nanocomposites have great potential application in high voltage industry field. Furthermore, it is still the focus of the study to improve these preparation methods, to discover new synthetic methods, and to prepare more homogeneous dispersion nanocomposites [27]. With the deepening of the research, people will be able to design and produce more excellent nanocomposites materials according to the actual needs.

\section{Summary}

This paper provides a review about the main four methods for preparing nanocomposites. The blending method is the most direct way for preparing polymer nanocomposites. However, nanoparticles are volatile, which need to disperse by the chemical action of fluid mechanics and surface physics. The intercalation method has excellent thermal stability and low cost, but it is limiting in some section, such as the filling materials in the monomer intercalation method generally have typical layered structure. The reaction condition of the in-situ dispersion polymerization method is mild and the nanoparticles dispersed uniformly, however this method is merely applicable for colloidal particles including metals. The reaction conditions of the sol-gel method are mild and controllable, but the precursors are expensive and toxic, and the production cycle is too long. This paper can provide beneficial references for the research and application of polymer nanocomposites in high voltage equipment.

\section{Acknowledgments}

This research was financially supported by the Application of Filled Resin-based Composite Insulating Materials in High Voltage Field, Scientific Research of Kunming Metallurgy College (NO. 2015XJYB003). 


\section{References}

[1] Z. M. Dang, J. K. Yuan, J. W. Zha, T. Zhou, S. T. Li, G. H. Hu, Fundamentals, processes and applications of high-permittivity polymer matrix composites, Prog. Mater. Sci. 57 (2012) 660-723. [2] X. M. Wang, W. P. Li, L. H. Luo, Z. Fang, J. Zhang, Y. J. Zhu, High dielectric constant and superparamagnetic polymer-based nanocomposites induced by percolation effect, Appl. Polym. Sci. 125 (2012) 2711-2715.

[3] T. Tanaka, T. Imai, Advances in nanodielectric materials over the past 50 years, IEEE Electr. Insul. M. 29 (2013) 10-23.

[4] L. W. Tang, H. Y. Chien, T. S. Yeong, C. Y. An, W. J. Li, C. Z. Hua, Synthesis of new nanocrystal-polymer nanocompositeas the electron acceptor in polymer bulk heterojunction solarcells, Eur. Polym. J. 46 (2010) 634-642.

[5] A. Kutvonen, G. Rossi, T. A. Nissila, Correlations between mechanical, structural, and dynamical properties of polymer nanocomposites, Phys. Rev. E 4 (2012) 1-6.

[6] T. Tanaka, A. Bulinski, J. Castellon, M. Frechette, S. Gubanski, J. Kindersberger, G. C. Montanari, M. Nagao, P. Morshuis, Y. Tanaka, Dielectric properties of XLPE/SiO2 nanocomposites based on CIGRE WG D1.24 cooperative test results, IEEE T. Dielect. El. In. 18 (2011) 1484-1517.

[7] K. S. Shah, R. C. Jain, V. Shrinet, A. K. Singh, D. P. Bharambe, High density polyethylene (HDPE) clay nanocomposite for dielectric applications, IEEE T. Dielect. El. In. 16 (2009) 853-861.

[8] T. Toshikatsu, M. Yasuaki, U. Katsumi, Partial discharge endurance of epoxy/SiC nanocomposite, 2008 IEEE Conference on Electrical Insulation and Dielectric Phenomena 1 (2008)

[9] M. Pramanik, S. K. Srivastav, B. K. Samantaray, A. K. Bhowmick, Rubber-clay nanocomposite by solution blending, J. Appl. Polym. Sci. 87 (2003) 2216-2220.

[10]H. U. Ko, A. John, S. Mun, J. Im, J. Kim, Preparation and characterization of cellulose-ZnO nanolayer film by blending Method, macromol. Res. 23 (2015) 814-818.

[11]C. Zhou, G. Z. Liang, A. J. Gu. Fabrication of variable frequency motors using polyesterimide-hybridized resins and hyperbranched polysiloxane coated nano-TiO2, J. Mater. Sci. 50 (2015) 7314-7325.

[12] Azizurrahaman, A. K. Jha, M. J. Akhtar, Thermal and microwave dielectric properties of $\mathrm{Cu}$ /polyethylene oxide composite powder prepared by mechanical blending method, Adv. Powder Technol. 26 (2015) 1281-1286.

[13]M. Herrero, S. M. Gallegos, F. M. Labajos, V. Rives, Layered double hydroxide/polyethylene terephthalate nanocomposites. Influence of the intercalated $\mathrm{LDH}$ anion and the type of polymerization heating method, J. Solid State Chem. 184 (2011) 2862-2869.

[14]R. Clement, Ion exchange intercalation into the MPS3 layered compounds: Design of hybrid nanocomposites with unusual magnetic, electrical and non linear optical properties, Polym. Mater. Sci. Eng. 70 (1993) 227-230.

[15]S. Mallakpour, M. Khani, Composites of Semiaromatic Poly (Amide-Ester-Imide) Based on Bioactive Diacid and Oragnomodified Nanoclay Produced by Solution Intercalation Method: Thermal and Morphological Study, Polym-Plast. Technol. 54 (2015) 541-547.

[16]Y. H. Chu, M. Yamagishi, Z. M. Wang, H. Kanoh, T. Hirotsu, Synthesis of nanoporous graphite-derived carbon/TiO2-SiO2 composites by a mechanochemical intercalation method, Micropor. Mesopor. Mat. 118 (2009) 496-502. 
[17]W. Xu, M. Ge, P. He, Nonisothermal crystallization kinetics of polyoxymethylene/ montmorillonite nanocomposite, J. Appl. Polym. Sci. 28 (2001) 2281-2289.

[18]J. Ren, Y. Liu, X. Z. Tang, Preparation and Properties of Polymeric Organic-inorganic Nanocomposites, Journal of building materials 7 (2004) 58-65.

[19]A. Ahmad, S. Milad, M. Saeedeh, K. Mohammadreza, Enhanced thermal properties of poly (ethylene tetrasulfide) via expanded graphite incorporation by in situ polymerization method, High Performance Polymers 25 (2013) 576-583.

[20]L. Ren, J. Zhao, S. J. Wang, B. Z. Han, Z. M. Dang, Dielectric and magnetic properties of Fe@FexOy/epoxy resin nanocomposites as high-performance electromagnetic insulating materials, Compos. Sci. Technol. 114 (2015) 57-63.

[21]A. m. Xie, F. Wu, Z. H. Xu, M. Y. Wang, In situ preparation of ultralight three-dimensional polypyrrole/nano SiO2 composite aerogels with enhanced electromagnetic absorption, Compos. Sci. Technol. 117 (2015) 32-38.

[22]E. Amerio, M. Sangermano, G. Malucelli, A. Priola, B. Voit, Preparation and characterization of hybrid nanocomposite coatings by photopolymerization and solgel Process, Polymer 46 (2005) 11241-11246.

[23]J. Y. Wen, G. L. Wildes, Organic/inorganic hybrid network materials by the sol-gel approach, Chem. Mater. 8 (1996) 1667-1681.

[24]T. Tanaka, G. C. Montanari, R. Muhaupt, Polymer nanocomposites as dielectrics and electrical insulation-perspectives for processing technologies, material characterization and future applications, IEEE T. Dielect. El. In. 11 (2004) 763-784.

[25]C. F. Kuan, W. H. Yen, C. H. Chen, S. M. Yuen, H. C. Kuan, C. L. Chiang, Synthesis, characterization, flame retardance and thermal properties of halogen-free expandable graphite/PMMA composites prepared from sol-gel method, Polym. Degrad. Stabil. 93 (2008) 13571363.

[26]L. Ma, Y. Yang, C. Y. He, Z. X. Jia, X. Y. Liu, J. Q. Qin, In situ preparation and characterization of polyimide/silica composite hemispheres by inverse aqueous emulsion technique and sol-gel method, Colloid Polym. Sci. 293 (2015) 1281-1287.

[27] M. Amin1, M. Ali, Polymer nanocomposites for high voltage outdoor insulation applications, Rev. Adv. Mater. Sci. 40 (2015) 276-294.

[28]W. Yan, Z. J. Han, B. T. Phung, F. Faupel, K. Ostrikov, High-Voltage Insulation OrganicInorganic Nanocomposites by Plasma Polymerization, Materials 7 (2014) 563-575.

[29]T. Tanaka, Y. Ohki, M. Ochi, M. Harad, T. Imai, Enhanced partial discharge resistance of epoxy/clay nanocomposite prepared by newly developed organic modification and solubilization methods, IEEE T. Dielect. El. In. 15 (2008) 81-89.

[30]S. Raetzke, J. Kindersberger, Role of Interphase on the Resistance to High-voltage Arcing, on Tracking and Erosion of Silicone/SiO2 Nanocomposites, IEEE T. Dielect. El. In. 17 (2010) 607-614. 